\title{
Application of damage locating vectors approach on monitoring shock isolation system
}

\author{
Quang Tuyen Le ${ }^{1}$, Chiung Shiann Huang ${ }^{1}$, and Wei Chih $\mathrm{Su}^{2, *}$ \\ ${ }^{1}$ Department of Civil Engineering, National Chiao Tung University, Hsinchu 30076, Taiwan \\ ${ }^{2}$ National Center for High-performance Computing, National Applied Research Laboratories, Hsinchu 30076, Taiwan
}

\begin{abstract}
Structural damage detection based on the changes of dynamic properties is a major topic for structural health monitoring. In this paper, efforts are made to extend the flexibility-based damage localization methods, especially the damage locating vectors (DLVs) method, to the case of earthquake vibration, where the finite element model and mass matrices are not available. First, a new method using continuous Cauchy wavelet transform (CCWT) and ARX (autoregressive with exogenous input) model is applied to identify the modal parameters of a five-storey steel frame with seismic base isolation system LRB from its simulated acceleration responses under $10 \%$ and $100 \%$ of Chi-Chi Earthquake excitation (Taiwan, 1999). The DLVs, which determined from the change of flexibility matrix between two cases, are then used to monitor the shock isolation device in the structure through a weighted relative displacement index (WRDI). The proposed scheme is also proved to be superior to mode shape based methods (MAC, COMAC) in monitoring shock isolation system.
\end{abstract}

\section{Introduction}

In recent years, beside the popular applications in signal processing, wavelet transform also has demonstrated its excellent analysis capabilities in time-frequency domain that made many applications in system identification research. Narrow into the field of continuous wavelet transform, there are some studies really helpful for identifying modal parameters. Schoenwald[1] applied the continuous wavelet transform to the equation of motion of a single degree freedom system and identified the parameters in the equation of motion. Gouttebroze and Lardies[2] processed vibration responses of structures using Morlet mother wavelet and estimated natural frequencies and damping ratios of structures. Lardies \& Gouttebroze[3] practiced their wavelet-based identification technique[2] to randomdec signatures of a TV tower. Huang \& $\mathrm{Su}[4]$ proposed a procedure combining continuous wavelet transform with time series model ARX to estimate modal parameters of a structure from its free vibration responses and seismic responses using different mother wavelets (Shannon wavelet, Meyer wavelet, Morlet wavelet and Haar wavelet).

Approaches based on the change of flexibility formed an important group of methods for structural damage detection[5,6]. Bernal[7] recently proposed a damage locating vectors (DLV) method for damage localization, and this technique was experimentally studied by Gao \& Spencer[8]. The generalized flexibility matrix change to detect the location and extent of structural damage has been introduced by Li et al.[9]. A method based on best achievable flexibility change with capability in detecting the location and extent of structural damage has also been presented by Yang \& Sun[10]. The flexibility matrix and strain energy concepts of a structure have been used by Nobahari \& Seyedpoor[11] in order to introduce a damage indicator for locating structural damage.

In this paper, a new method using continuous Cauchy wavelet transform (CCWT) and ARX (autoregressive with exogenous input) model is applied to identify the modal parameters of a five-storey steel frame with seismic base isolation system LRB from its simulated acceleration responses under $10 \%$ and $100 \%$ of Chi-Chi Earthquake excitation (Taiwan, 1999). The DLV's vectors, which determined from the change of flexibility matrix between two cases, are then used to monitor the shock isolation device in the structure through a weighted relative displacement index. The proposed scheme is also proved to be superior to mode shape based methods (MAC, COMAC) in monitoring shock isolation system.

\section{Methodology}

\subsection{Identification of modal parameters using CCWT and ARX model}

The dynamical responses of a linear structure are described by the equation of motion

$$
[M]\{\ddot{x}\}+[C]\{\dot{x}\}+[K]\{x\}=\{f\}
$$

where $[M],[C]$, and $[K]$ are the mass, damping and stiffness matrices of the structure system, respectively; $\{\ddot{x}\},\{\dot{x}\}$, and $\{x\}$ are the acceleration, velocity and displacement response vectors of the system, and $\{f\}$ is

\footnotetext{
Corresponding author: wichcv86@gmail.com
} 
the input force vector. Equation 1 can be accurately discretized by the impulse invariant transformation as [12]:

$$
\begin{aligned}
\{z(t)\}= & \sum_{i=1}^{I}\left[\Phi_{i}\right]\{z(t-i \Delta t)\} \\
& +\sum_{j=0}^{J}\left[\Theta_{j}\right]\{\overline{\mathbf{f}}(t-j \Delta t)\}
\end{aligned}
$$

where $\Delta t$ that represents the time increment.

$$
\begin{aligned}
& \{z(t)\}=\left\{\begin{array}{l}
\ddot{x}(t) \\
\dot{x}(t)
\end{array}\right\} \\
& \{z(t-i \Delta t)\}=\left\{\begin{array}{l}
\ddot{x}(t-i \Delta t) \\
\dot{x}(t-i \Delta t)
\end{array}\right\}
\end{aligned}
$$

I, J denote the lags of output and input, respectively; $\left[\Phi_{i}\right],\left[\Theta_{j}\right]$ are coefficient matrices related to $[\mathrm{M}],[\mathrm{C}]$, $[\mathrm{K}]$ that need to be determined. Equation 2 is very similar to the time series ARX model with multiple variables.

The continuous Cauchy wavelet transform of a real signal of finite energy is

$$
W_{\psi} f(b, a)=\frac{1}{\sqrt{a}} \int_{-\infty}^{+\infty} f(t) \psi_{n}^{*}\left(\frac{t-b}{a}\right) d t
$$

where the standard Cauchy mother wavelet with order $n$ is defined as:

$$
\psi_{n}(t)=\left(\frac{i}{t+i}\right)^{n+1}
$$

Treating $z(t-i \Delta t)$ and $\overline{\mathbf{f}}(t-j \Delta t)$ as vector functions, and applying the continuous Cauchy wavelet transform to Equation 2 yields

$$
\begin{aligned}
W_{\psi} z(a, \bar{b})= & \sum_{i=1}^{I}\left[\Phi_{i}\right] W_{\psi} z(a, \bar{b}-i) \\
& +\sum_{j=0}^{J}\left[\Theta_{j}\right] W_{\psi} \bar{f}(a, \bar{b}-i)
\end{aligned}
$$

where the translation parameter $b$ is set to be $\bar{b} \Delta t$. Constructing Equation 6 for different $\bar{b}$ and rearranging the resulting equations gives

$$
\left[Z^{(0)}\right]=[\hat{C}]\left[\begin{array}{l}
{[Z]} \\
{[F]}
\end{array}\right]
$$

where

$$
[\hat{C}]=\left[[\Phi]_{,} \quad[\Phi]_{2} \ldots[\Phi]_{,} \quad[\Theta]_{0} \quad[\Theta]_{1} \ldots[\Theta]_{,}\right]
$$

$$
\begin{aligned}
& {[Z]=\left[\left[Z^{(1)}\right]^{T} \quad\left[Z^{(2)}\right]^{T} \ldots\left[Z^{(I)}\right]^{T}\right]^{T}} \\
& {[F]=\left[\left[F^{(1)}\right]^{T} \quad\left[F^{(2)}\right]^{T} \ldots\left[F^{(J)}\right]^{T}\right]^{T}}
\end{aligned}
$$

The coefficient matrix $[\hat{C}]$ is calculated by the least squares or the generalized inverse approaches. Hence:

$$
[\hat{C}]=\left[Z^{(0)}\right]\left[\begin{array}{l}
{[Z]} \\
{[F]}
\end{array}\right]^{+}
$$

where the superscript "+" denotes the generalized inverse operation. By adopting the concept behind the Ibrahim time domain system identification technique, Huang[12] proved that the modal parameters (natural frequencies, damping ratios and mode shapes) of the structure can be estimated from the eigenvalues and eigenvectors of $[\mathrm{G}]$, which is constructed from coefficient matrices as:

$$
[G]=\left[\begin{array}{cccccc}
0 & I & 0 & 0 & \ldots & 0 \\
0 & 0 & I & 0 & \ldots & 0 \\
\vdots & & & & & \\
{\left[\Phi_{I}\right]} & {\left[\Phi_{I-1}\right]} & & & & {\left[\Phi_{1}\right]}
\end{array}\right]
$$

where $I$ is an $1 * 1$ unit matrix, and 1 is the dimension of measured $\{z(t)\}$.

\subsection{DLVs approach}

A simple damage localization method was proposed by Pandey and Biswas (1994), which consists in calculating the flexibility change matrix:

$$
\Delta F=F_{d}-F_{i}
$$

where $F_{\mathrm{i}}$ and $F_{\mathrm{d}}$ are the flexibility matrices for the intact and the damaged cases, respectively. The DLVs can be obtained from singular value decomposition (SVD) of $\Delta F$. Each of DLVs is then applied to the intact model of structure. The stress $\sigma_{i}$ in each structural element is calculated and a normalized cumulative stress (nsi) is obtained. If an element has zero normalized cumulative stress, then it is a possible candidate of damage.

In practice, the normalized cumulative stress induce by DLVs in the damaged elements may not be exactly zero due to noise and uncertainties. According to Hooke's law, under static condition:

$$
\{f\}=K\{\Delta x\}
$$

where $\mathrm{K}$ and $\{\Delta x\}$, respectively, are the stiffness matrix and relative displacement of the system subjected by force $\{f\}$. Equation 12 can be written as: 


$$
\{\Delta y\}=\hat{F}\{g\}
$$

where

$$
\begin{gathered}
\{\Delta y\}=M^{1 / 2}\{\Delta x\} \\
\hat{F}=M^{1 / 2} K^{-1}\left(M^{1 / 2}\right)^{T} ;\{g\}=\left(M^{-1 / 2}\right)^{T}\{f\}
\end{gathered}
$$

In the flexibility based-damage detection method, the DLVs are applied to structure as static force at the sensor locations to check whether stress area get zero value will be considered as damage position. So Equation 13 can be rewritten by:

$$
\{\Delta y\}=\hat{F}_{d}\{L\}
$$

where $\hat{F}_{d}$ is the flexibility matrix of damage structure, and $\{L\}$ is the damage locating vectors (DLVs).

Define the normalized relative displacement index of jth element as

$$
n r d i_{j}=\left|\Delta y_{j+1}-\Delta y_{j}\right| /\left|\Delta y_{j+1}-\Delta y_{j}\right|_{\max }
$$

one can compute a weighted relative displacement index (WRDI) as

$$
W R D I=\sum_{j=1}^{q} \gamma_{j} n r d i_{j}
$$

where $\gamma_{j}=$ weights; $\mathrm{q}$ is the number of DLVs. Taking $\{P D\}$ as the set of potentially damaged elements one has

$$
P D=\{\text { elements } \mid W R D I \leq t o l\}
$$

In this paper, we select $\gamma=1$, tol $=0.1(\mathrm{WRDI})_{\max }$ for a simple approach.

\section{Application to shock isolation system}

To confirm the accuracy and effectiveness of the proposed approach, SAP2000 was used to build up a finite element model of five-storey steel and simulate this frame (see Fig. 1) subjected to base excitations of the 10\% 1999 Chi-Chi earthquake (Case 1) and 100\% 1999 Chi-Chi earthquake (Case 2).

The five-storey steel frame under consideration was $3 \mathrm{~m}$ long, $2 \mathrm{~m}$ wide and $15 \mathrm{~m}$ high. A seismic isolation system LRB is set up near base at the bottom of first storey to reduce the effect of earthquake excitation onto the structure. The simulation was conducted for $5 \%$ of the modal damping ratio. Assume plates were fixed on each floor, such that the total mass of steel frame was approximately 3.3516 tons. All types of data were saved at a sampling rate of $250 \mathrm{~Hz}$.

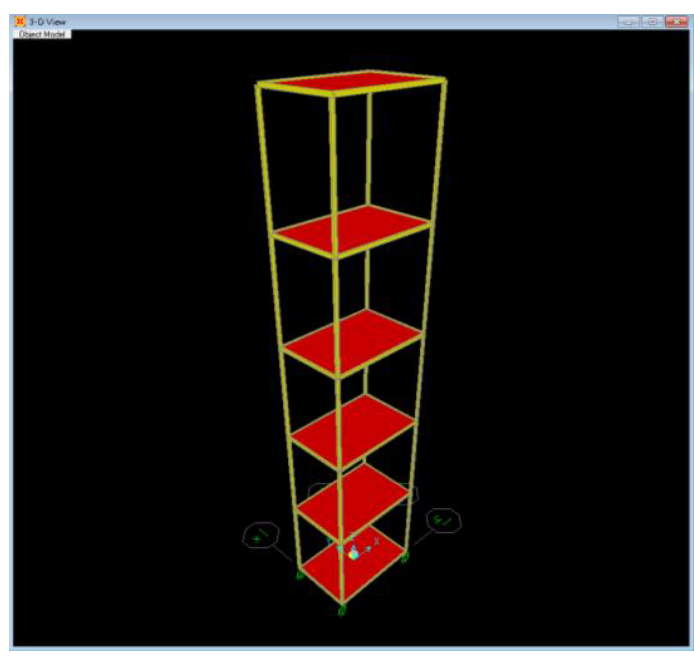

Fig. 1. Schematic diagram of a five-story shear frame.

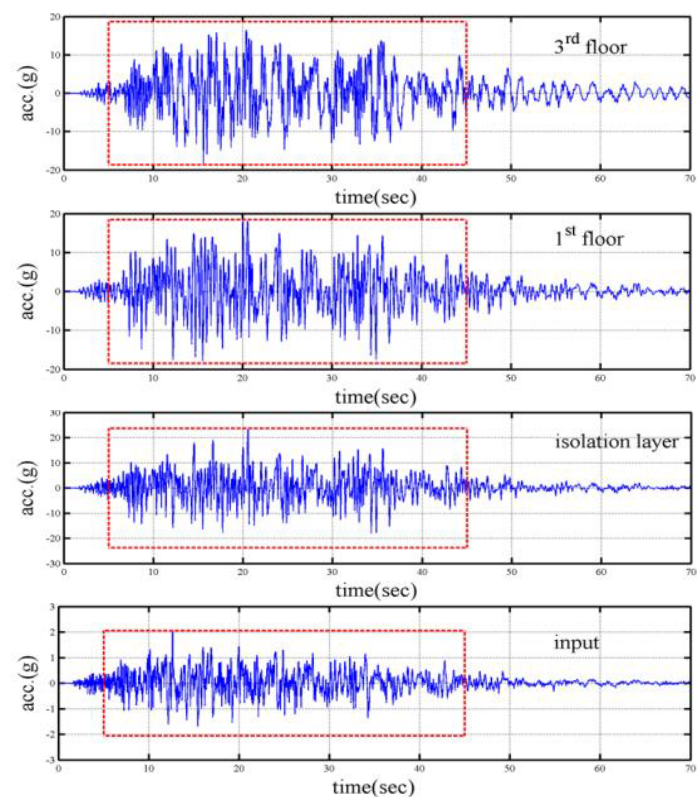

Fig. 2. Time history of base excitation and simulated acceleration responses of some floors of the frame in Case 1

By using full measurement responses of the frame in CCWT method, Table 1 and Table 2 respectively show the identified modal parameters of frame at two case obtained using Cauchy wavelets with different values of " $n+1$ " and scale "a" corresponding to the judged natural frequencies.

Table 1. The identified modal parameters of frame under $10 \%$ 1999 Chi-Chi earthquake (Case 1)

\begin{tabular}{ccccccc} 
Mode & $1^{\text {st }}$ & $2^{\text {nd }}$ & $3^{\text {rd }}$ & $4^{\text {th }}$ & $5^{\text {th }}$ & $6^{\text {th }}$ \\
\hline$f_{n}(\mathrm{~Hz})$ & 0.77 & 2.30 & 3.84 & 5.52 & 7.35 & 8.72 \\
\hline$\xi(\%)$ & 0.05 & 0.05 & 0.05 & 0.05 & 0.05 & 0.05 \\
\hline \multirow{4}{*}{ Modal shapes } & 1.00 & 1.00 & 0.85 & -0.66 & -0.47 & -0.21 \\
& 0.92 & 0.37 & -0.33 & 0.83 & 1.00 & 0.56 \\
& 0.56 & -0.45 & -0.98 & 0.33 & -0.82 & -0.86 \\
& 0.31 & -0.94 & -0.23 & -0.94 & -0.01 & 1.00 \\
& 0.14 & -0.56 & 1.00 & -0.03 & 0.83 & -0.98 \\
& & & & & -0.90 & 0.65
\end{tabular}


Table 2. The identified modal parameters of frame under $100 \%$ 1999 Chi-Chi earthquake (Case 2)

\begin{tabular}{|c|c|c|c|c|c|c|}
\hline Mode & $1^{\mathrm{st}}$ & $2^{\text {nd }}$ & $3^{\text {rd }}$ & $4^{\text {th }}$ & $5^{\text {th }}$ & $6^{\text {th }}$ \\
\hline$f_{n}(\mathrm{~Hz})$ & 0.75 & 2.23 & 3.66 & 5.39 & 7.19 & 8.62 \\
\hline$\xi(\%)$ & 0.21 & 0.11 & 0.06 & 0.04 & 0.04 & 0.04 \\
\hline \multirow{6}{*}{ Modal shapes } & 1.00 & -0.99 & 0.82 & -0.73 & -0.49 & -0.23 \\
\hline & 0.93 & -0.41 & -0.24 & 0.87 & 1.00 & 0.61 \\
\hline & 0.79 & 0.40 & -0.92 & 0.44 & -0.73 & -0.90 \\
\hline & 0.61 & 0.96 & -0.38 & -0.98 & -0.17 & 1.00 \\
\hline & 0.41 & 1.00 & 0.68 & -0.22 & 0.93 & -0.91 \\
\hline & 0.29 & 0.72 & 1.00 & 1.00 & -0.70 & 0.49 \\
\hline
\end{tabular}

The index of modal assurance criterion (MAC)[13] and coordinate modal assurance criterion (COMAC) [14] was computed to indicate the correlation between any two mode shapes of interest:

$$
\begin{gathered}
\operatorname{MAC}(j)=\frac{\left|\sum_{i=1}^{n} \varphi_{i j, 1} \varphi_{i j, 2}\right|^{2}}{\left[\left(\sum_{i=1}^{n} \varphi_{i j, 1}^{2}\right)\left(\sum_{i=1}^{n} \varphi_{i j, 2}^{2}\right)\right]} \\
\operatorname{COMAC}(i)=\frac{\left|\sum_{j=1}^{m} \varphi_{i j, 1} \varphi_{i j, 2}\right|^{2}}{\left[\left(\sum_{j=1}^{m} \varphi_{i j, 1}^{2}\right)\left(\sum_{j=1}^{m} \varphi_{i j, 2}^{2}\right)\right]}
\end{gathered}
$$

to compare the identified mode shapes of undamaged frame in Case 1 with damaged frame in Case $2, \varphi_{i j, 1}$ and $\varphi_{i j, 2}$ represent the deformation of the ith degree of freedom in the jth identified mode shapes, respectively.

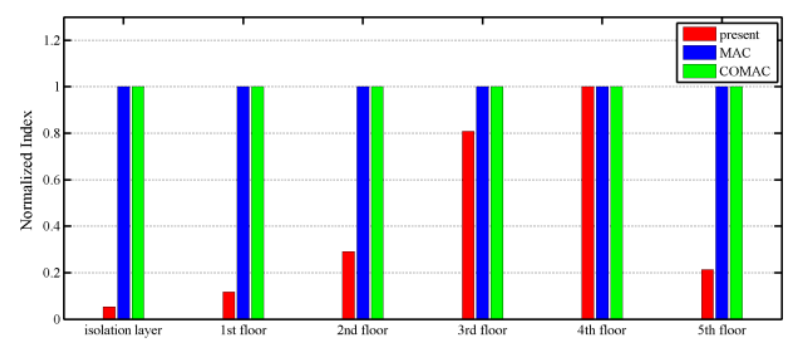

Figure 3. Normalized index

When frame was subjected to $10 \% 1999$ Chi-Chi earthquake excitation, no nonlinear behavior were observed, and it was treated as a reference structure. Based on result of WRDI index, we got the value of potential damage $\mathrm{PD}=1$ which happened at the isolation layer of frame. This PD value correlated to the "present" diagram in Figure 3 and showed that there is damage in the seismic isolation layer only after frame subjected to 100\% 1999 Chi-Chi earthquake excitation. The LRB system showed nonlinear behavior and the flexibility increase. This result also proved that the isolation system LRB had performed well its mission to absorb the power of the strong earthquake excitation onto the frame.

Figure 3 clearly demonstrates that the proposed approach is superior to MAC and COMAC methods in identifying the floor of the structure whose properties differ from those of the corresponding floor in the reference structure.

\section{Concluding remark}

The work develop a simple and efficient flexibility based approach, especially the damage locating vectors (DLVs) method, for monitoring the seismic isolation system on a structure. To demonstrate the feasibility of proposed method for actual application, the procedure was applied to a five-storey shear frame (with isolation system LRB set up at first floor) which subjected to $10 \%$ and $100 \%$ of 1999 Chi-Chi earthquake excitation. The proposed approach was validated by successfully identify the damage position through processing measured responses. The isolation system showed nonlinear behavior and absorbed the power of strong excitation of earthquake. Comparing the results obtained by the proposed approach with MAC and COMAC indexes revealed that the present approach is substantially superior to both in monitoring shock isolation system.

\section{Acknowledgement}

The authors would like to thank the Ministry of Science and Technology of Taiwan for financially supporting this research under Contract No. MOST 105-2622-M492-001-CC2.

\section{References}

1. D. A. Schoenwald, In proceedings of the $32^{\text {nd }}$ IEEE Conference on Decision and Control, (1993)

2. S. Gouttebroze \& J. Lardies, Mechanics Research Communications 28, 5 (2001)

3. J. Lardies \& S. Gouttebroze, International Journal of Mechanical Sciences 44, 11 (2002)

4. C.S. Huang \& W. C. Su Mechanical System and Signal Processing 21, 4 (2007)

5. S.W. Doebling \& C.R. Farrar, Report LA-13070MS. Los Alamos National Laboratory (1996)

6. A.K. Pandey \& M. Biswas, Journal of Sound and Vibration, 169 (1994)

7. D. Bernal, Journal of Engineering Mechanics, 128 (2002)

8. Y. Gao \& B.F. Spencer, Proceedings of the $1^{\text {st }}$ International Workshop on Advanced Smart Materials and Smart Structures Technology (2004)

9. J. Li, B. Wu, O.C. Zeng \& C.W. Lim, Journal of Sound and Vibration 329, 22 (2010)

10. Q.W. Yang \& B.X. Sun, Applied Mathematical Modelling, 35, 10 (2011)

11. M. Nobabari \& S.M. Seyedpoor, Structural Engineering and Mechanics, 46, 2 (2013)

12. C.S. Huang, Journal of Sound and Vibration, 241, 3 (2001)

13. R.L. Allemang \& D.L. Brown, Proceeding of the $1^{\text {st }}$ International Modal Analysis Conference, (1983)

14. N.A. Lieven \& D.J. Ewins, In Proceedings of the $6^{\text {th }}$ International Modal Analysis Conference, (1988) 